\title{
Early Gastric Cancer following Gastrojejunostomy without Gastrectomy
}

\author{
TOHRU UMEZU, JINRYO TAKEDA, TAKAHO TANAKA, KIKUO KOUFUJI, \\ KEN HASHIMOTO AND TERUO KAKEGAWA \\ Department of Surgery, Kurume University School of Medicine, \\ Kurume, 830 Japan
}

Received for publication June 19, 1991

\begin{abstract}
Summary: Two rare cases involving distal gastrectomies for gastric cancer after an earlier gastrojejunostomies without gastrectomy for duodenal or gastric stricture due to a duodenal or pre-pyloric ulcer are reported. The time interval between the initial gastrojejunostomy and the resection of the distal stomach with gastrojejunal stoma were 17 and 36 years. One patient underwent reconstruction by Billroth $I$ and the other by Roux-en- $Y$ end-to-side gastrojejunostomies, both retrocolically without any peri or postoperative complications. The histological classification was confirmed for both as type IIc mucosal early gastric cancer. Only 6 other primary early gastric cancers following gastrojejunostomies without gastrectomy have been reported in the Japanese literature.
\end{abstract}

Key words : early gastric cancer - gastrojejunostomy — endoscopy — stomadistal gastrectomy - postoperative complication

\section{Introduction}

Any increase in the number of patients with remnant gastric cancer following partial gastrectomy and gastroenterostomy for benign gastroduodenal disease is of special interest since the overall incidence of primary gastric cancer is declining in Japan. There is convincing evidence that the incidence of duodenogastric reflux is of importance for inducing changes in the postoperative gastric mucosa, and this has been studied experimentally and clinically. However, gastric cancer following gastroenterostomy for benign gastroduodenal diseases is rare in Japan. Moreover, early gastric cancer following gastrojejunostomy without gastrectomy has been reported in only 6 cases in the Japanese literature. Two new and very rare cases of early gastric cancer following gastrojejunostomy will be described with particular attention to the early diagnosis and surgical management.

\section{Patients and Methods}

Case 1

A 54 year-old male underwent gastrojejunostomy for duodenal stricture due to a duodenal ulcer in 1966. Seventeen years later, he experienced epigastric pain and hematoemesis. He was hospitalized as an emergency patient and examined by upper gastroenteral endoscopy. $\mathrm{He}$ was diagnosed as having a large bleeding gastric ulcer in the gastric angle and probably also early gastric cancer type IIc in the gastric angle.

He underwent a distal gastrectomy with 
resection of the previously-anastomosed jejunal stoma with an $R_{1}$ dissection of the lymph nodes of Group 1. A reconstruction by Billroth II (B-II) end to side gastrojejunostomy retrocolically, as an emergency procedure, was also performed. There was a large bleeding gastric ulcer in the gastric angle, $3.7 \times 2.5$ $\mathrm{cm}$ in size. Histologically, $5 \mathrm{~mm}$ of a mucosal cancer (m-cancer) of a poorly differentiated adenocarcinoma (por) type was confirmed near the U1-IV gastric ulcer. Lymph node metastasis was pathologically negative. The patient was discharged from the hospital 30 days after the operation without any postoperative complications and is now disease-free, 8 years postoperatively.

\section{Case 2}

A 56 year-old male underwent gastrojejunostomy without gastrectomy for prepyloric stricture due to gastric ulcers in 1953. Thirty-six years later, he experienced epigastric pain. He was examined by upper gastroenteral roentgenography, endoscopy and endoscopic biopsies, and was diagnosed as having an early gastric cancer and a gastric ulcer in the gastrojejunal stoma. Fig. 1 shows a roentgenogram of the suspected early gastric cancer of a superficial depressed type IIc and the gastric ulcers near the stoma. A biopsy specimen demonstrated that the gas tric cancer was a signet-ring cell carcinoma (sig).

The patients underwent a distal gastrectomy with resection of the previouslyanastomosed jejunal stoma with $\mathrm{R}_{3}$ dissection of the lymph nodes of Groups 1 , 2 and 3 and underwent reconstruction by Roux-en - Y end to side gastrojejunostomy retrocolically. The resected specimen was a type IIc early gastric cancer of $3.5 \times$ $3.0 \mathrm{~cm}$ in size near the stoma and gastric ulcer (Fig. 2). Fig. 3 shows the pathological findings of the mucosal ( $\mathrm{m}$-cancer) signet-ring cell carcinoma which infil-

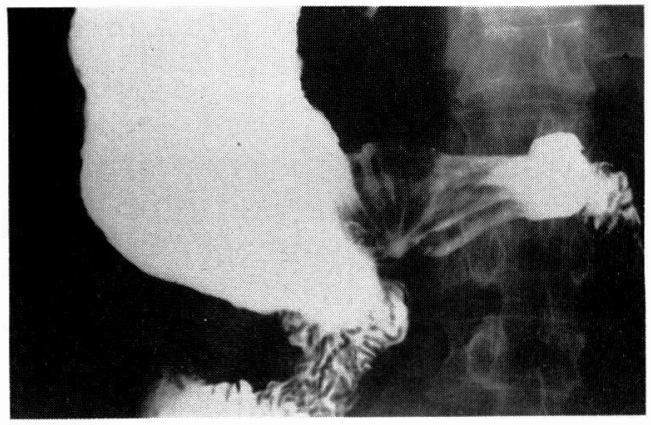

Fig. 1. Roentgenogram of the suspected early gastric cancer of a superficial depres ses type Ic and a gastric ulcer near the stoma.
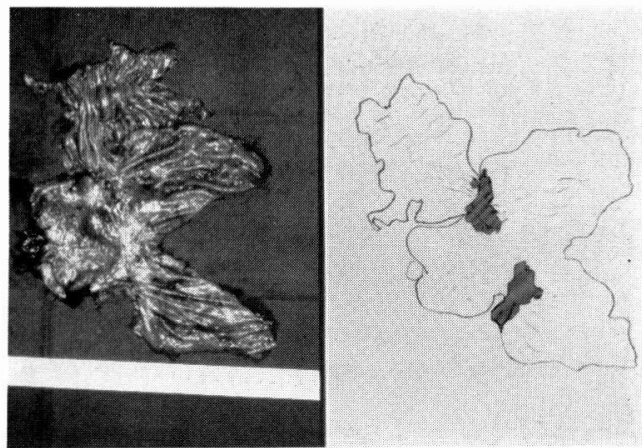

Fig. 2. The resected specimen was a type Ilc early gastric cancer, $3.5 \times 3.0 \mathrm{~cm}$ in size, near the stoma and gastric ulcer.

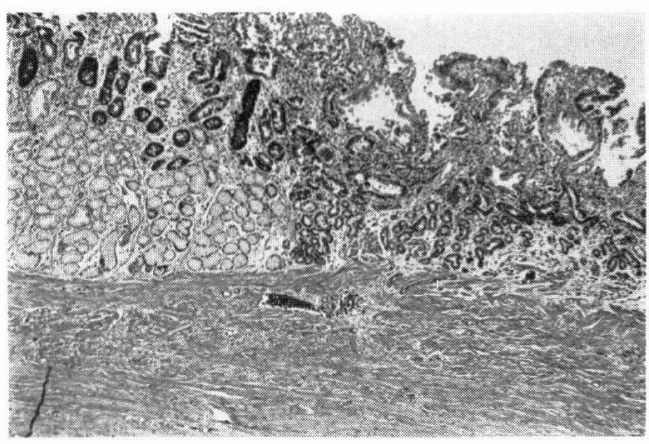

Fig. 3. Pathological specimen of the mucosal ( $\mathrm{m}$-cancer) signet-ring cell carcinoma infiltrating across the stoma to the anastomosis of the jejunum. $(\times 100)$ 
trated across the stoma to the anastomoses of the jejunum. Lymph node metastasis $(n=57)$ was negative, pathologically. The patient was discharged from the hospital 21 days after the operation without any postoperative complications and is now disease-free, 3 years and 5 months postoperatively.

\section{Discussion}

There have been some studies on primary gastric remnant-stump cancers following partial gastrectomies for benign gastroduodenal disease, especially in other countries (Saegesser and James, 1972; Nicholls, 1974; Sons and Borchard, 1987). The incidence of primary gastric remnant-stump cancer following partial gastrectomy is now increasing, even in Japan (Kidokoro et al. 1985), despite a declining overall incidence of gastric cancer in Japan. However, a primary gastric cancer following gastroenterostomy without gastrectomy for a benign gastroduodenal disease is rare, especially in Japan, and those rare cases that have been reported have been mainly described at the time of diagnosis. This study describes two very rare cases of early gas- tric cancers following gastrojejunostomy without gastric resection, with special attention to the early diagnosis and operative techniques for successful management.

Early gastric cancer is defined as a cancer with invasion that is limited either to the mucosa (m-cancer) or the sbumucosa (sm-cancer), but not extending deeper histologically, with or without lymph node metastasis, according to the Japanese Research Society for Gastric Cancer (1981).

Table 1 shows a summary of the 8 known cases (including the present 2) of early gastric cancers following gastrojejunostomy without gastrectomy for a benign gastroduodenal disease. There were 7 males and 1 female with a mean age of 56 years. The original diseases were gastric (G-) ulcers in the three, duodenal (D-) ulcers in the four, duodenal diverticulitis (D-Dive) in one and congenital pyloric stenosis (Congenital) in one patient. The time interval between the initial operation and the second cancer operation ranged from 10 to 51 years (mean: 26 years). Three (38\%) of the 8 cancers were located in the gastrojejunal stoma and $4(50 \%)$ were in the lesser curvature (Min.) including 1 in the gastric

TABLE 1

Early gastric cancers following gastrojejunostomies: Japanes ecases

\begin{tabular}{|c|c|c|c|c|c|c|c|c|}
\hline \multirow[b]{2}{*}{ Author } & \multirow[b]{2}{*}{ Year } & \multicolumn{4}{|c|}{ Patient } & \multicolumn{3}{|c|}{ Cancer } \\
\hline & & Age & Sex & $\begin{array}{l}\text { Initial } \\
\text { Disease }\end{array}$ & $\begin{array}{l}\text { Time } \\
\text { Interval }\end{array}$ & Location & Operation & Classification \\
\hline Hattori & 1969 & 67 & M & G-Ulcer & 22 & A-Min & Distal & $\mathbb{I I}+\Pi \mathrm{Ia}(\mathrm{m})$ \\
\hline Nishidoi & 1983 & 25 & $\mathrm{~F}$ & Congenital & 20 & A & Total & IIa (tub2) \\
\hline Ishii & 1983 & 50 & M & G-Ulcer & 20 & Stoma & Distal & IIc (sig) \\
\hline Inui & 1984 & 47 & M & D-Ulcer & 47 & A-Min & Distal & II c (tub2) \\
\hline Kurokawa & 1986 & 73 & M & D-Dive & 10 & A-Min & Distal & IIa (tub1) \\
\hline Shimizu & 1988 & 72 & M & G-Ulcer & 51 & Stoma & Distal & II $c+$ III (sig) \\
\hline \multirow[t]{2}{*}{ Our } & 1983 & 54 & M & D-Ulcer & 17 & M-Angle & Distal & IIc (por) (m) \\
\hline & 1988 & 56 & $\mathrm{M}$ & G-Ulcer & 36 & Stoma & Distal & IIc $(\mathrm{sig})(\mathrm{m})$ \\
\hline
\end{tabular}


angle. Four $(50 \%)$ of the 8 were in the antrum.

Viste et al. (1986) reported that among 819 patients with stump cancer, $41(5 \%)$ patients had undergone an earlier gastroenteroanastomy without gastrectomy. Sons and Bouchard (1987) reported that the average time interval to the detected manifestation of cancer was 22.6 years after B-II, 23. 4 years after B-I, and 34. 4 years after gastroentero-anastomosis without resection. In the 8 known cases of early gastric cancer following gastrojejunostomy, the time interval was 26 years.

In this report, 2 patients with early gastric cancer had presented with the preoperative clinical symptom of abdominal pain, and one had hematemesis. However, these symptoms were considered to probably be due to the presence of deep and wide gastric ulcer.

Before endoscopic examinations, they were checked by X-ray, indicating the presence of gastric ulcers. One also had a suspected early gastric cancer. The diagnosis of early gastric cancer by $\mathrm{X}$-ray is generally difficult because of the deformity of the stomach, reflux gastritis and the incidence of gastric ulcers following gastrojejunostomy. In this study, both early gastric cancers were diagnosed by preoperative endoscopic examinations. However, an expert endoscopist is required for such an examination because of the inherent difficulty in detecting early cancer due to reflux gastritis related to gastroentero-anastomosis.

For the 8 known cases, the cancer location was mainly in the antrum and around the stoma, therefore, distal gastrectomy and groups 1 and 2 lymph node dissections were sufficient for the early cancers. However, a combined resection of the anastomosed jejunum with a wide margin is required because of the known high incidence of clinical lymph node metastasis of the mesenterium by cancers in the stoma and because of a newly confirmed lymph route from the stomach to the jejunum across the stoma (Kodama, 1989).

It can be concluded that to detect early cases careful examination is required by endoscopy especially near the stoma with follow - up multiple biopsies for confirmation. Advanced cancers should be managed with an aggressive extended gastrectomy, including removal of the anastomosed jejunum with a wide margin, of the perigastric juxta regional lymph nodes and of the para-aortic lymph nodes with occasional combined resection of the colon when infiltrated for curative treatment.

\section{References}

Japanese Research Society for Gastric Cancer. The general rules for the gastric cancer study in surgery and pathology. (1981). Jpn. J. Surg. 11, 127-139.

Kodama, I. (1989). Experimental study on lymphatic flow of the remnant stomach. Jpn. J. Gastroenterol. Surg. 22, 1172-1181.

Kidokoro, T., Hayashida, Y. and URABe, M. (1985). Long-term surgical results of carcinoma of the gastric remnant: A statistical analysis of 613 patients from 98 institutions. World J. Surg. 9, 966-971.

NicholLs, J.C. (1974). Carcinoma of the stomach following partial gastrectomy for benign gastroduodenal lesions. Br. J. Surg. 61, 244249.

SAegesser, F. and James, D. (1972). Cancer of the gastric stump after partial gastrectomy (Billroth II principle) for ulcer. Cancer 29, 11501159.

Sons, H.U. and Borchard, F. (1987). Gastric carcinoma after surgical treatment for benign ulcer disease. Some pathologic-anatomic aspects. Int. Surg. 72, 222-226.

Viste, A., Eide, G.E., Real, C., Glatre, E. and SoReID, O. (1986). Cancer of the gastric stump: Analysis of 819 patients and comparison with other stomach cancer. 